\title{
Functional characterization of the promoter region of bovine Neuropeptide $Y$ (NPY) gene
}

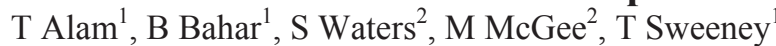

${ }^{1}$ Cell and Molecular Biology Lab, Veterinary Science Centre, University College Dublin, Dublin, Ireland, ${ }^{2}$ Grange Beef Research Centre, Dunsany, Co Meath, Ireland

Email: tanweerbio@gmail.com

Introduction Neuropeptide is a mammalian neurotransmitter produced by the brain which plays a vital role in energy homeostasis of mammals, including the bovine (Kaiyala et al., 1995). Neuropeptide Y (NPY) is a potent orexigenic agent that increases appetite and feed intake, and therefore is a potential candidate for enhancing feed energy utilization in cattle (White, 1993). Single nucleotide polymorphisms (SNPs) characterized in the promoter region of the bovine NPY gene (Bahar and Sweeney, 2009) have potential for genetic selection of animals with high feed energy efficiency. However, the molecular mechanisms underlying the regulation of bovine $N P Y$ gene expression by its promoter region is currently unknown. The objectives of this research were to characterize the transcriptional activities of the bovine $N P Y$ gene and identify the minimal promoter required for basal activity of $N P Y$ gene in vitro.

Materials and methods For identification of minimal promoter of the bovine $N P Y$ gene, $-1019 \mathrm{~kb}$ to +1 (reference to the transcription start site) region of the bovine NPY gene (GeneBank: AY491054) was targeted. Promoter deletion constructs of 38-1019 nt length (Figure 1) were made in a firefly luciferase expression vector system (pGL 4.17, Promega Corp.). The in vitro promoter transcription efficiency of the promoter constructs were performed using a mouse 3T3-L1 host cells system. Firefly luciferase activity in the cell lysate was measured using a luminometer. Total protein concentration in the cell lysate was estimated using the BCA protein assay kit (Thermo Scientific). Firefly luciferase activity was normalised against the total protein. Data was analysed using the GraphPad prism 5 and are reported as the mean of the three independent assay \pm standard error of the mean.

Results The in vitro transcriptional activities of the different deletion constructs of the bovine $N P Y$ promoter are shown in Figure 2. It is evident from the promoter activity data that there was an increase in the promoter activity with increase in the promoter length upto-134 nt. However, there was a substantial decrease in the promoter activity for the promoter length 148 to $-289 \mathrm{nt}$ indicating the presence of suppressor elements in this region. Again, promoter activity increases upto -703 nt. Taken together, these results suggested that the sequence up to -134 nt are sufficient for basal promoter activity, however, for the maximal promoter activity the region upto $-703 \mathrm{nt}$ is essential.

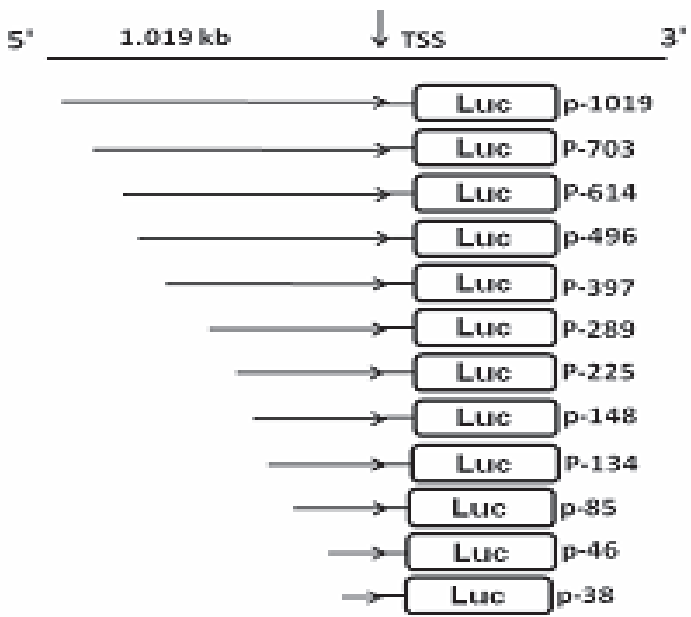

Figure 1 Schematic representation of the 12 bovine NPY promoter deletion constructs. The number (eg. $\mathrm{p}-1019)$ indicates the relative nucleotide position from the transcriptional start site (TSS; Position +1) and hence the construct size (eg. 1019n).

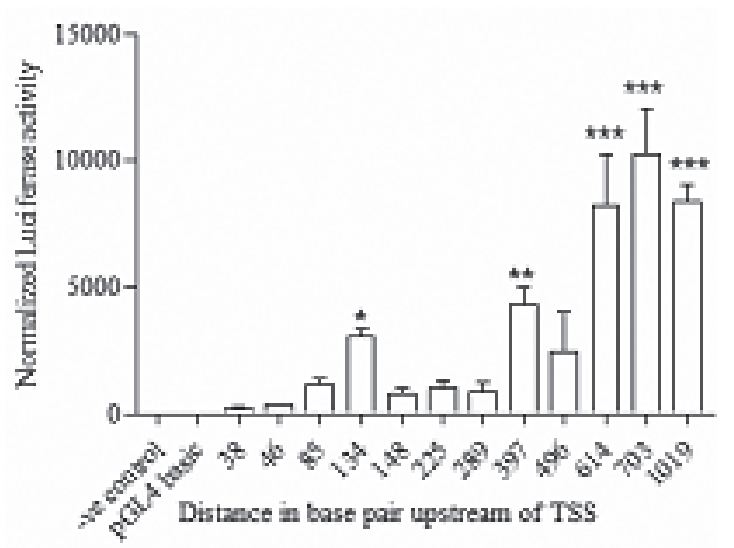

Figure 2 Luciferase activity of the bovine $N P Y$ promoter deletion constructs. Luciferase activity was normalized against total protein.

Data points shown are the means \pm SE. $P<0.05$ considered significant

Conclusion In vitro promoter activity of the deletion constructs ranging from 38 nt to 1019 nt of the bovine NPY promoter demonstrated that a promoter length up to $-134 \mathrm{nt}$ is sufficient for the basal promoter activity, while a length up to -703 is actually required for maximal promoter activity. The SNPs present in the various regions of the bovine NPY promoter especially those affecting the transcription factor binding sites and those in the enhancer and repressor regions have the potential to affect neuropeptide $\mathrm{Y}$ mediated feed energy utilization in cattle.

Acknowledgements This research was supported by a Teagasc Walsh Fellowship.

\section{References}

Bahar, B. and Sweeney, T. 2008. BMC Genetics 9, 91

Kaiyala, K.J., Woods, S.C., and Schwartz, M.W.1995. American Journal of Clinical Nutrition 62,1123S-34S

White, J.D.1993. Regulatory Peptides 49, 93-107 